\title{
Perampanel for Refractory Status Epilepticus... Another Tool in the Armamentarium
}

\author{
Sara Hocker
}

C 2019 Springer Science+Business Media, LLC, part of Springer Nature and Neurocritical Care Society

Despite a dramatic increase in research over the past decade, patients with refractory status epilepticus (RSE) continue to have high mortality and survivors experience significant disability. RSE is defined as status epilepticus which continues despite treatment with a benzodiazepine and a second antiseizure drug (ASD). Its treatment remains a relatively 'evidence-free zone', and is frequently difficult to abort, making any additional treatment options relevant and potentially important. In this issue of Neurocritical Care, Ho et al. [1] reported a retrospective analysis of 67 consecutive patients with RSE, 22 of whom were treated with perampanel. Perampanel, a novel ASD, acts as a noncompetitive $\alpha$-amino-3-hydroxy5-methyl-4-isoxazolepropionic acid (AMPA) receptor antagonist, and has the effect of reducing glutamatemediated postsynaptic excitation. In animal models, perampanel has been shown to successfully terminate benzodiazepine RSE when GABAergic inhibition decreases and the ratio of AMPA receptor subunits changes $[2,3]$. Recent case series have reported on a total of 41 patients treated with perampanel for predominantly nonconvulsive or focal motor RSE; however, they used variable criteria to define drug responders and reported conflicting results [4-6].

The aim of this descriptive study was to further describe the effectiveness of perampanel for different status epilepticus subtypes. Drug responders were defined as being clinically and electrographically seizure

\footnotetext{
*Correspondence: hocker.sara@mayo.edu Division of Critical Care Neurology, Mayo Clinic College of Medicine, 200 First Street SW, Rochester, MN 55905, USA
}

This comment refers to the article available at https://doi.org/10.1007/ s12028-019-00704-9. free within 4 days with perampanel being the last ASD used, and not developing recurrence of status epilepticus during hospitalization. They report that 8 of 22 (35\%) patients treated with perampanel were responders. While the authors also conclude that the drug may be more effective for focal motor and convulsive than for nonconvulsive subtypes, there was, in fact, no difference between subtypes in responders or nonresponders. To quantify the drug's effect on ictal and interictal electroencephalogram (EEG), EEG recordings were reviewed at day 0 (before perampanel), day $1(6-24 \mathrm{~h})$, day 2 (24-48 h), and days 3-4 (48-96 h). Notably, interictal epileptiform discharge frequency and average ictal time percentage decreased gradually with time after initiation of perampanel therapy, achieving maximal benefit by the third to fourth day of treatment.

Response to perampanel in this case series is higher than in published studies. This is made more impressive when noting the median number of ASDs used to control RSE was significantly higher in the 22 patients receiving perampanel [5] compared with the 45 who did not [3], and increased percentage of super RSE (59 vs $24 ; p=0.002$ ). It adds to a growing literature suggesting efficacy in RSE. The drug is particularly attractive (as was levetiracetam before it), because of the novel mechanism of action involving AMPA compared with other commonly used ASDs which preferentially target GABA (phenobarbital and sodium valproate), and sodium channels (phenytoin, sodium valproate, and lacosamide) [7], and because of its favorable safety profile [8].

Strengths of the study include detailed clinical and electrographic information and information on a sizable number of patients, specifically regarding the timing of effect. This study has important limitations. The most substantial is that termination of seizures cannot 
be attributed to perampanel alone as patients had been necessarily treated with multiple other drugs and where possible, for the cause of seizures. As the authors mention, response rate is likely overinflated due to the higher proportion of patients with convulsive status epilepticus compared with prior series. Additionally, the retrospective design, nonstandardized timing of initiation, dosing, and prior treatment limit interpretation of both efficacy and generalizability.

Perampanel may be a reasonable option for treatment of RSE. Clinicians may need to exercise patience before determining that a patient is not responding to perampanel. While the limitations of this study are extensive, we should not be dogmatic in the interpretation as there is little evidence to guide treatment at the refractory stage of status epilepticus. Thus, perampanel can be considered to be yet another tool in an ever expanding armamentarium for a notoriously challenging disease.

\section{Source of support}

None.

\section{Conflicts of interest}

The authors declare that they have no conflict of interest.

\section{Publisher's Note}

Springer Nature remains neutral with regard to jurisdictional claims in published maps and institutional affiliations.

\section{References}

1. Ho CJ, Lin CH, Lu YT, Shih FY, Hsu CW, Tsai WC, Tsai MH. Perampanel treatment for refractory status epilepticus in a neurological intensive care unit. Neurocrit Care. 2019. https://doi.org/10.1007/s12028-019-00704-9.

2. Leo A, Giovannini G, Russo E, Meletti S. The role of AMPA receptors and their antagonists in status epilepticus. Epilepsia. 2018;59(6):1098-108.

3. Rajasekaran K, Todorovic M, Kapur J. Calcium-permeable AMPA receptors are expressed in a rodent model of status epilepticus. Ann Neurol. 2012;72(1):91-102.

4. Redecker J, Wittstock M, Benecke R, Rosche J. Efficacy of perampanel in refractory nonconvulsive status epilepticus and simple partial status epilepticus. Epilepsy Behav. 2015;45:176-9.

5. Rohracher A, Hofler J, Kalss G, et al. Perampanel in patients with refractory and super-refractory status epilepticus in a neurological intensive care unit. Epilepsy Behav. 2015;49:354-8.

6. Rohracher A, Kalss G, Neuray C, et al. Perampanel in patients with refractory and super-refractory status epilepticus in a neurological intensive care unit: a single-center audit of 30 patients. Epilepsia. 2018;59(52):234-42.

7. Sills GJ. Mechanisms of action of antiepileptic drugs. In: Sander JW, Walker MC, Smalls JE, editors. Epilepsy 2011. From science to society. A practical guide to epilepsy (Chapter 25)

8. Serratosa JM, Villanueva V, Kerling F, Kasper BS. Safety and tolerability of perampanel: a review of clinical trial data. Acta Neurol Scand Suppl. 2013;197:30-5. 